\title{
The Shooting Method Applied to a Cyclic Inequality
}

\author{
By B. A. Troesch
}

Abstract. It is known that the cyclic sum

$$
S_{n}(\mathbf{x})=\sum_{i=1}^{i=n} x_{i} /\left(x_{i+1}+x_{i+2}\right),
$$

where $x_{n+1}=x_{1}, x_{n+2}=x_{2}, x_{i} \geqslant 0,\left(x_{i+1}+x_{i+2}\right)>0$, can be made smaller than $n / 2$ for $n \geqslant 24$. The value of $\lambda(n)=\lim \inf (n \rightarrow \infty) S_{n} / n$ is investigated by the shooting method for two-point boundary value problems, and the analytical result $\lim (n \rightarrow \infty) \lambda(n) \leqslant 0.49457$ is proved. The inherent difficulty in a straightforward minimization of $S_{n}(\mathbf{x})$ is mentioned.

1. Introduction. Many results are known about the cyclic sum

$$
S_{n}(\mathrm{x})=\sum_{i=1}^{i=n} x_{i} /\left(x_{i+1}+x_{i+2}\right)
$$

formed with the $n$ components of a vector $\mathbf{x}$, where $x_{i+n}=x_{i}, x_{i} \geqslant 0$, and $x_{i+1}+$ $x_{i+2}>0$ for all $i$ (see [9] for the origin of the problem and [5] for the results and the history of the inequality connected with this sum). If we introduce

$$
\sigma(n)=S_{n}(\mathbf{x}) / n, \quad \lambda(n)=\inf \sigma(n),
$$

the infimum being taken over all admissible vectors $\mathbf{x}$, then $\lambda(n) \leqslant 1 / 2$, because $x_{i}=1$ for all $i$ is an admissible choice. Nowosad [6, p. 463] has proved that $\lambda(n)=1 / 2$ for $n \leqslant 10$; on the other hand, a general relation [2] and counterexamples for $n=14$ and $n=25$ (see [8] and the references given there) show that $\lambda(n)<1 / 2$ for even $n \geqslant 14$ and for all $n \geqslant 24$.

In [7] Rankin proved the existence of the limit

$$
\lambda=\lim _{n \rightarrow \infty} \lambda(n) \leqslant \lambda(n), \quad n \geqslant 2,
$$

and also showed that $\lambda>0.304$. Later Diananda [3] improved this lower bound to

$$
\lambda(n)>0.4612 \ldots .
$$

Upper bounds are simply obtained from numerical examples, e.g.,

$$
\lambda \leqslant \sigma(111)=0.49656
$$

given by Daykin [1]

Received March 19, 1979.

AMS (MOS) subject classifications (1970). Primary 10E20, 26A86; Secondary 34-04, 34B1 5.

Key words and phrases. Cyclic inequality, shooting method, minimization. 
In this note we investigate a possible behavior of $\sigma(n)$ for large $n$ and obtain by elementary means (Section 5) an improvement on the upper bound for $\lambda$ :

$$
\lambda \leqslant 0.494566817
$$

The vector $\mathbf{x}$ chosen for this result is suggested by a quite effective numerical approach, described in Section 2, namely a recursive approach, based on the well-known shooting method for two-point boundary value problems in ordinary differential equations.

Throughout, we restrict ourselves to a certain class of $x_{i}$ 's: it is assumed that the $x_{i}$ 's contain only one string of contiguous alternate zero terms (see, for instance, Figure 2). Although there is no proof that this class of $x_{i}$ 's leads indeed to the lowest $\sigma(n)$ for large $n$, we note that Daykin's solution for $n=111$ [1] has also this structure. The upper bound of Eq. (3) is independent of this assumption.

The computational results are discussed in Section 3. There is a heuristic argument concerning the value of $\lambda$ presented in Section 4 . It leads to the conjecture that

$$
\lambda>0.49438 \text {. }
$$

Although the problem of the cyclic inequality is essentially a minimization problem, a direct minimization would lead to serious numerical difficulties. Some comments on this question are presented in the Appendix.

2. Recursion Relation. We are attempting to make the cyclic sum as small as possible. Clearly, no vector $\mathbf{x}$ with two vanishing consecutive components is a contender $[6$, p. 446]. A necessary condition for a minimum is therefore

$$
x_{i} \partial S / \partial x_{i}=0 \text { for all } i
$$

i.e.,

$$
x_{i}\left\{\frac{x_{i-2}}{\left(x_{i-1}+x_{i}\right)^{2}}+\frac{x_{i-1}}{\left(x_{i}+x_{i+1}\right)^{2}}-\frac{1}{x_{i+1}+x_{i+2}}\right\}=0 .
$$

It follows that either $x_{i}=0$ or

$$
x_{i+2}=-x_{i+1}+1 /\left\{\frac{x_{i-2}}{\left(x_{i-1}+x_{i}\right)^{2}}+\frac{x_{i-1}}{\left(x_{i}+x_{i+1}\right)^{2}}\right\} .
$$

Now, it is permissible to use the normalization $x_{1}=1$ and to set $x_{2}=0$, since Nowosad [6] has shown that among the minimizing vectors there is always a vector with at least one zero component. If we make some choice for $x_{3}$ and $x_{4}$, the values of $x_{i+2}(i \geqslant 3)$ can be computed recursively from Eq. (5), provided that $x_{i} \neq 0$. For a fixed $n$, the guesses $x_{3}$ and $x_{4}$ would then be adjusted until Eq. (5) were also satisfied for $i=n-1$ and $i=n$. However, we are not trying to minimize $\sigma(n)$ for any particular $n$; we are simply aiming at making $n$ as large as possible. The recurrence relation (5) is therefore used until the two end conditions are essentially satisfied. This turns out to be quite easy, if we make use of our assumption stated above: if, say, $x_{k+2}$ is the first term to become zero or negative, then $x_{k+2}$ and all subsequent alternate terms, $x_{k+4}, x_{k+6}, \ldots, x_{n}, x_{2}$ are also assumed to vanish. 
Good starting guesses for $x_{3}$ and $x_{4}$ are not hard to obtain. From the known results for odd $n$ from 25 to 31 and for even $n$ from 14 to 26, and 38 [8] an extrapolation to large $n$ indicates that $x_{3} \doteq 1.17, x_{4} \doteq 0.15$ is a good choice. Daykin's values for $n=111$ [1] are about the same. (Actually, this initial guess is an interpolation between even and odd $n$ values (see Figure 1).)

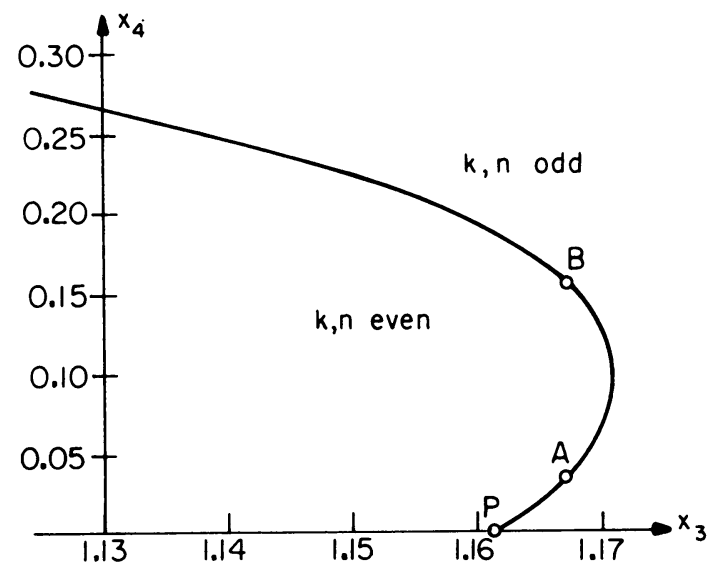

FIGURE 1

Curve of initial conditions $x_{3}, x_{4}$ for minimum of $\sigma(n)$.

$$
P\left(x_{3}=1.1613, x_{4}=0\right) \text {. }
$$

From the initial guesses the values $x_{5}, x_{6}, \ldots$ are computed in turn until the $k$ is reached for which $x_{k+2} \leqslant 0$. Then, with $x_{k+2 j}, j=1,2, \ldots$, all vanishing, Eq. (5) leads to $(n-k+2) / 2$ equal ratios

$$
b=x_{k+3} / x_{k+1}=x_{k+5} / x_{k+3}=\cdots=x_{1} / x_{n-1} \doteq x_{3} / x_{1} .
$$

Therefore, we have

$$
x_{1} / x_{k+1}=b^{(n-k) / 2}=\left(x_{3} / x_{1}\right)^{(n-k) / 2},
$$

and

$$
(n-k) / 2 \doteq m=-\log x_{k+1} / \log x_{3}
$$

Normally, $m$ will not turn out to be an integer as required (for this reason the last equal sign in Eq. (6) is only approximate), but it furnishes an approximation to a suitable integer $M$. In the computation, several values were tried for the determination of the $x_{k+2 j+1}$, and it was found that the best $M$, leading to the smallest $\sigma(n)$, exceeds $m$ by about 2 or 3 . Hence, for $n=k+2 M$ we obtain an admissible upper bound

$$
\lambda(n) \leqslant \sigma(n)=S_{n}(\mathbf{x}) / n \text {. }
$$

The shooting computation is very fast, even in double precision (28-digit numbers). Since we attempt to make $n$ large, $k$ should also be as large as possible (ideally, infinite). In order to approach the "boundary condition at infinity", $x_{k+2}=0$, we adopt a bisection method based on the following observation: it is well known that alternate $x_{i}$ values combine to a curve (see, for instance, Figures 2 and 5); if the 
curves intersect, then $k$ will be odd, if they do not intersect, then $k$ is even. Therefore, we keep $x_{4}$ fixed and bisect $x_{3}$ on the parity of $k$, a procedure which is both safe and simple. (In exploratory computations the curve in Figure 1 was determined both by a vertical and by a horizontal search.)

3. Results of the Numerical Computation. The results of the numerical computations are shown in Figure 1, and one might hope that on this curve a certain point would furnish a distinctly lowest $\sigma(n)$. But the results in Table 1 show that, for comparable $n$ values, this is not the case. The value of $\sigma(n)$ does not depend strongly on the initial conditions $x_{3}, x_{4}$, as long as they lie on the curve in Figure 1 to, say, nine significant digits. On the other hand, $\sigma(n)$ is quite well determined by $n$ or by $k$. Over a wide range of $k$ values we observe that $\sigma(n) \doteq \sigma(\infty)+c / k$, where $c=0.080$. If we extrapolate the data to $k \rightarrow \infty$, we obtain as the limit

$$
\lambda \leqslant \sigma(\infty)=0.49455 \pm 0.00005,
$$

in good agreement with Eq. (3) above.

It is conjectured that this represents indeed the true value of $\lambda$, even though its determination is based on the class of $x_{i}$ 's with only one contiguous string of zeros in alternate terms.

It is of interest to observe which $x_{i}$ 's lead to a small $\sigma(n)$ for large $n$, since this information is used in Section 5 below. First, we note that it makes a significant difference for small $n$, whether $n$ is odd or even. If we connect the two groups of alternate $x_{i}$ 's to form two curves, then these curves must intersect for odd $n$, whereas they need not intersect for even $n$. This means that for odd $n$ there is actually one curve closing on itself after $2 n$ steps. On the other hand, for even $n$ there are two curves of length $n$, each one closing on itself (see Figure 2). This would explain why the cyclic inequality is violated for odd $n>24$, but for even $n>12$, only half that number.

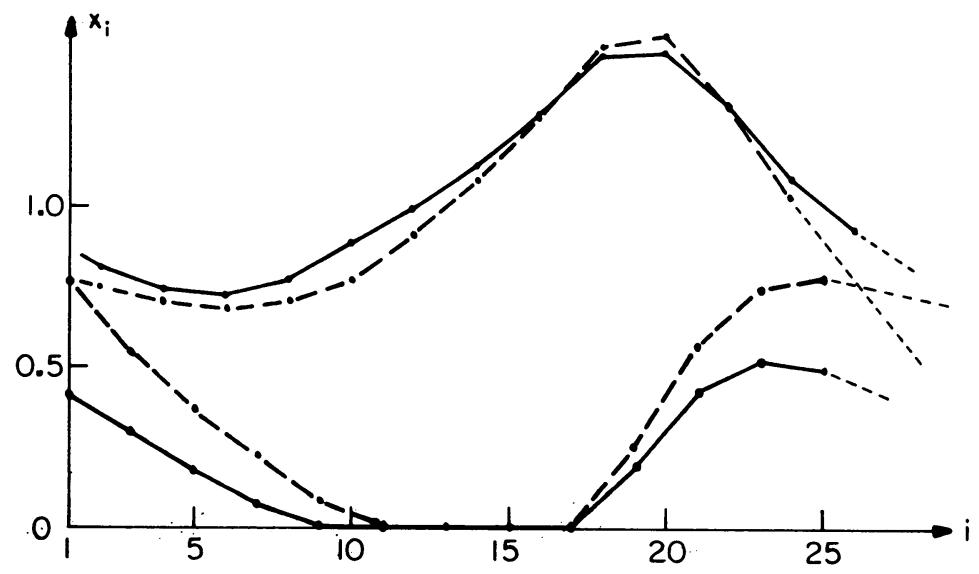

FIGURE 2

Basic difference between odd and even $n$

- for $n=26$ (noncrossing curves)

--- for $n=25$ (crossing curves). 
As $n$ gets larger, the distinction disappears gradually (see Table 1, where both odd and even entries appear). The two curves are shown, in part, in logarithmic scale in Figure 3. They flow together with the nearly constant ratio $a=x_{j+1} / x_{j}=0.90447$ (namely over 150 points to this accuracy), and then, after one curve dips to $x_{k+2} \leqslant$ 0 , the other curve rises with the constant ratio (see Eq. (6)) $b=x_{j+2} / x_{j}=1.16811$.

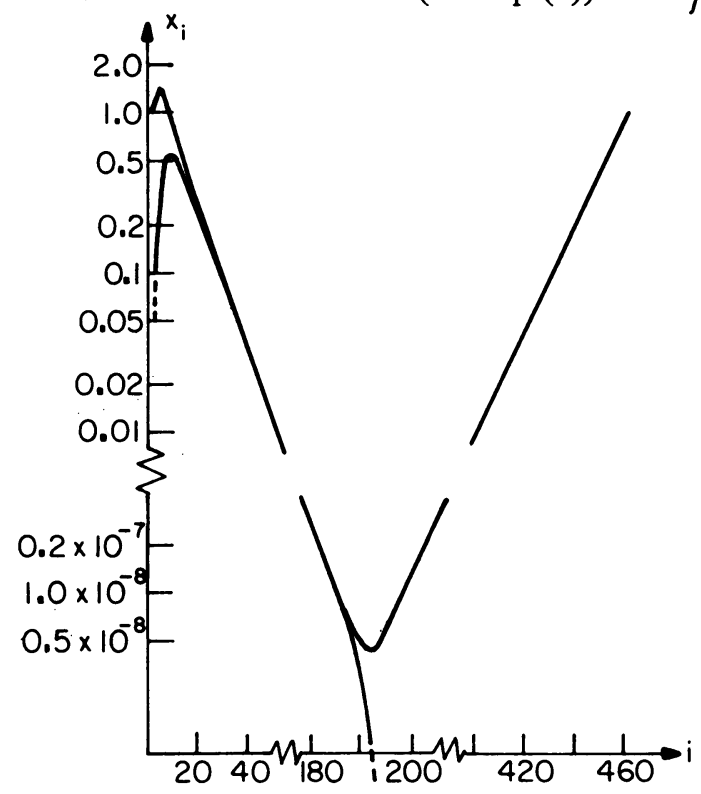

FIGURE 3

Values of $x_{i}$ for $x_{3}=1.168, x_{4}=0.1492$.

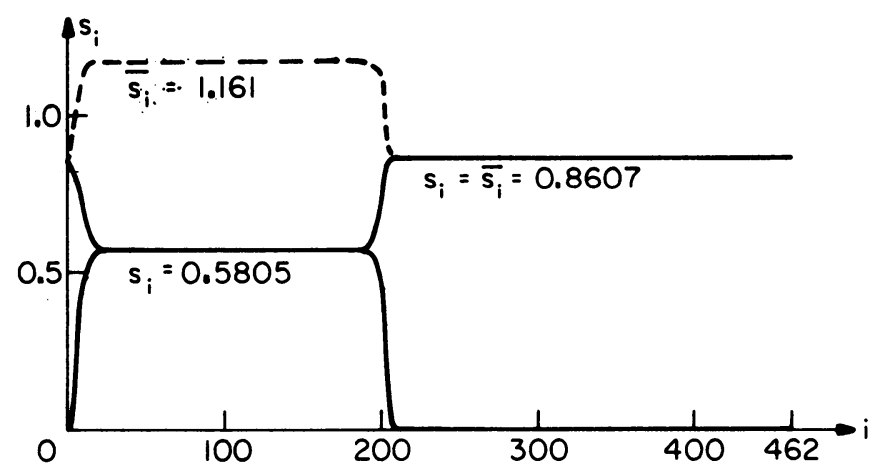

FIGURE 4

$$
\begin{aligned}
\text { Values of }-s_{i} & =x_{i} /\left(x_{i+1}+x_{i+2}\right), \\
----\bar{s}_{i} & =\left(s_{i-1}+2 s_{i}+s_{i+1}\right) / 2 .
\end{aligned}
$$

The terms $s_{i}=x_{i} /\left(x_{i+1}+x_{i+2}\right)$, again connected to form two curves, are shown in Figure 4, where, in addition, $\overline{s_{i}}=\left(s_{i-1}+2 s_{i}+s_{i+1}\right) / 2$ is also plotted. This leads to a single curve with two long stretches of constant values. Since $\sigma(n)=$ $S(\mathrm{x}) / n=(1 / 2 n) \Sigma \overline{s_{i}}$, it is clearly the second segment from $x_{k+2}$ up to $x_{2}$ which causes $\sigma(n)$ to become less than $1 / 2$. Furthermore, we confirm the empirical fact [8] 
that the $s_{i}$ exhibit a symmetry; for instance, in the case shown in Figure 4

$$
s_{105+j}=s_{105-j} \text { for all } j,
$$

although the $x_{i}$ are very asymmetric.

TABLE 1

Upper branch in Figure 1

\begin{tabular}{ccccr}
$x_{3}$ & $x_{4}$ & $k$ & $n$ & \multicolumn{1}{c}{$\sigma(n)$} \\
1.16700 & 0.1567 & 198 & 436 & 0.494849 \\
1.16800 & 0.1492 & 208 & 462 & 836 \\
1.16900 & 0.1403 & 196 & 424 & 854 \\
1.17000 & 0.1288 & 202 & 434 & 846 \\
1.17100 & 0.1083 & 197 & 419 & 856 \\
1.17114 & 0.0970 & 199 & 421 & 855 \\
& & & & 796 \\
1.16789 & 0.1502 & 244 & 538 & 785 \\
1.16789 & 0.1502 & 255 & 565 & 858 \\
Lower branch in Figure 1 & & & 858 \\
1.17103 & 0.0865 & 198 & 416 & 853 \\
1.17002 & 0.0646 & 197 & 417 & 856 \\
1.16901 & 0.0522 & 197 & 425 & 0.494859 \\
1.16801 & 0.0425 & 195 & 423 &
\end{tabular}

We have tacitly assumed that, as $i$ increases, the terms decrease until $x_{k+2} \leqslant 0$. However, sometimes both parts of the coalescing curves turn upwards. It then appears that the curves repeat the same pattern, only scaled down as shown in Figure 5. Since this situation did not lead to a smaller $\sigma(n)$ value in preliminary runs, the lower curve was set to zero at the minimum. Again, the symmetry in the $s_{i}$-curves in Figure 5 is rather striking.

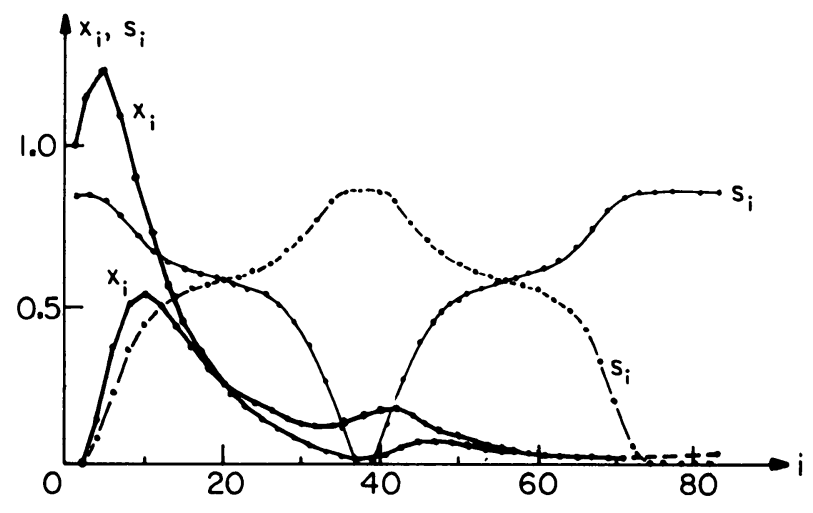

Figure 5 
The shape of the curve in Figure 1 of initial values $x_{3}$ and $x_{4}$ which lead to a large number of terms seems surprising: for one $x_{3}$ value there are two different $x_{4}$ values furnishing about the same $\sigma(n)$. But this is actually not so. If the $x_{i}$ 's are scaled and the numbering shifted by one, then the lower branch from $P$ on and the corresponding segment of the upper branch represent the same solution except for the first few points. This is shown by the curves (a) and (b) in Figure 6.

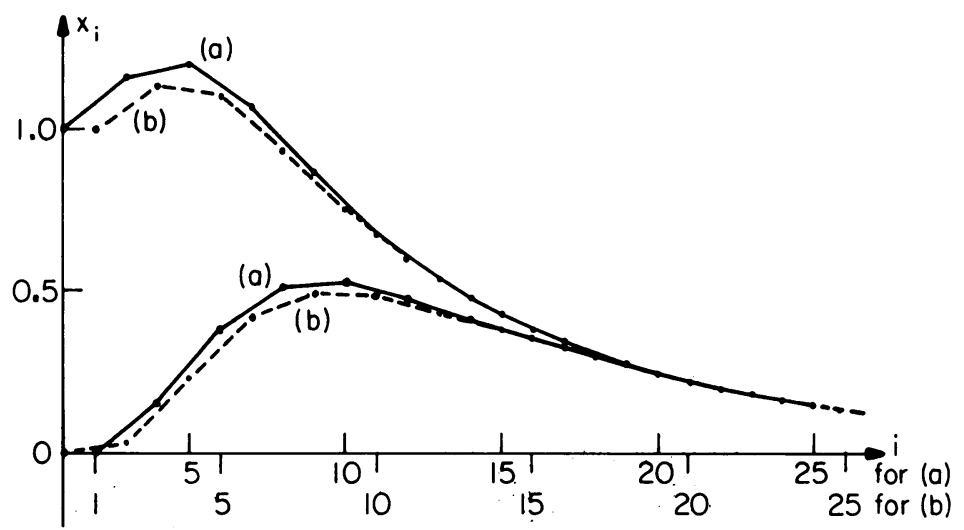

FiguRE 6

Comparison of upper branch (a) and lower branch (b) of Figure 1:

(a) $x_{3}=1.167, x_{4}=0.156$;

(b) $x_{3}=1.167, x_{4}=0.034$, but all $x_{i}$ scaled by $1 / 1.167$ and the subscripts shifted by one.

4. A Conjectured Lower Bound for $\lambda$. Upper bounds for $\lambda$ are obtained by exhibiting suitable examples for $\sigma(n)$. A heuristic argument for a lower bound for $\lambda$ can be based on the computations in Section 2. But the result would be conclusive only if the assumption about the $x_{i}$ 's having a single string of contiguous alternate zeros could be proved. In Section 2 we have inserted $(m-1)$ nonvanishing terms between $x_{k+1}$ and $x_{1}$ (see Eqs. (6) and (7)), so that $1 / x_{k+1}=b^{m}=x_{3}^{m}$, where we assume for simplicity that $m$ is an integer. If we denote the first part of the sum $S(x)$ by

$$
S_{0}=\sum_{i=1}^{i=k} x_{i} /\left(x_{i+1}+x_{i+2}\right)
$$

then $S(n)=S_{0}+m / b$.

Now we argue that for very large $n$ it should make no appreciable difference, if two more terms (one of them vanishing) are inserted; in other words, $\sigma(n+2)-\sigma(n)$ should go to zero for $n \rightarrow \infty$. Therefore, we insert two terms, set

$$
1 / x_{k+1}=\hat{b}^{(m+1)},
$$

where the new ratio is denoted by $\hat{b}$, and obtain

$$
S(n+2)=S_{0}+(m+1) / \hat{b}=S(n)+(m+1) / b^{m / m+1}-m / b .
$$

We assume that $S(n)$ corresponds to the infimum of $\sigma(n)$, so that 


$$
\sigma(n+2)-\lambda(n)=\frac{1}{n+2}\left\{(m+1) / b^{m / m+1}-m / b-2 \lambda(n)\right\} .
$$

There are all indications that this difference decays slower than $1 /(n+2)$, so that we obtain for $n \rightarrow \infty$

$$
\lambda=1 / 2 \lim _{m \rightarrow \infty}\left\{(m+1) / b^{m / m+1}-m / b\right\}
$$

or, setting $m+1=1 / z$ with $z \rightarrow 0$,

$$
\lambda=(1+\log b) / 2 b .
$$

Of course, $b=x_{3}$ is not exactly known, but the computation and Figure 1 indicate strongly that $x_{3} \leqslant 1.17114$. Hence, we conjecture that $\lambda \geqslant 0.494381$.

TABLE 2

$$
\begin{array}{ccccrrr}
b=x_{3} & 1.167 & 1.168 & 1.169 & 1.170 & 1.171 & 1.17114 \\
(1+\log b) / 2 b & 0.494617 & 0.494560 & 0.494503 & 0.494446 & 0.494389 & 0.494381
\end{array}
$$

5. An Upper Bound for $\lambda$. The argument just presented can be made independent of any computational result and it furnishes then an upper bound for $\lambda$. We exhibit a long vector $\mathrm{x}$ by choosing the $n=k+2 j-1$ vector components (with slightly changed numbering) as

$$
\begin{array}{ll}
x_{i}=a^{i-1} & \text { for } i=1,2, \ldots, k, \\
x_{i}=0 & \text { for } i=k+1, k+3, \ldots, k+2 j-1, \\
x_{i}=a^{k-1} b^{(i-k) / 2} & \text { for } i=k+2, k+4, \ldots, k+2 j-2 .
\end{array}
$$

The $(n+1)$ st term agrees with $x_{1}$, so that

$$
a^{k-1} b^{j}=1 \text { or } j=-(k-1) \log a / \log b
$$

and

$$
n=(k-1)(1-2 \log a / \log b) .
$$

For the cyclic sum we obtain

and

$$
S(\mathbf{x})=\frac{k-2}{a(1+a)}+\frac{1}{a}+\frac{j}{b}
$$

$$
\sigma(n)=\left\{\frac{1}{a(1+a)}-\frac{\log a}{b \log b}+\frac{1}{(k-1)(1+a)}\right\} /(1-2 \log a / \log b) .
$$

A straightforward minimization with respect to $a$ and $b$ and letting $k \rightarrow \infty$ leads to

$$
\begin{gathered}
b=a(1+a)^{2} /(1+2 a), \\
\log \left(\frac{b}{a^{2}}\right)=\log \left(\frac{(1+a)^{2}}{a(1+2 a)}\right)=\frac{1}{1+2 a},
\end{gathered}
$$


with the solution

$$
a=0.90447055, \quad b=1.167885645 .
$$

The values furnished by the numerical computation in Section 3 for $n=565$ are in close agreement.

From the equation

$$
\sigma(\infty)=\left\{\frac{\log b}{a(1+a)}-\frac{\log \bar{a}}{b}\right\} / \log \left(b / a^{2}\right)
$$

or, by eliminating $a$,

$$
\sigma(\infty)=(1+\log b) / 2 b,
$$

we obtain the result $\lambda \leqslant 0.494566817$.

It is not surprising that the equation for $\sigma(\infty)$ agrees with Eq. (9) above, since the underlying idea is the same, although here the value for $b$ is available.

Appendix. A Minimization. The pairs of initial conditions $x_{3}, x_{4}$ that lead to a small $\sigma(n)$ lie on the curve shown in Figure 1. Any values that deviate only slightly from the curve result in a considerable increase of $\sigma(n)$, so that the level curves form a canyon. The Figure 7 shows a cross section of it. The canyon walls are steep, but the bottom is very flat (cf. the results listed in Table 1).
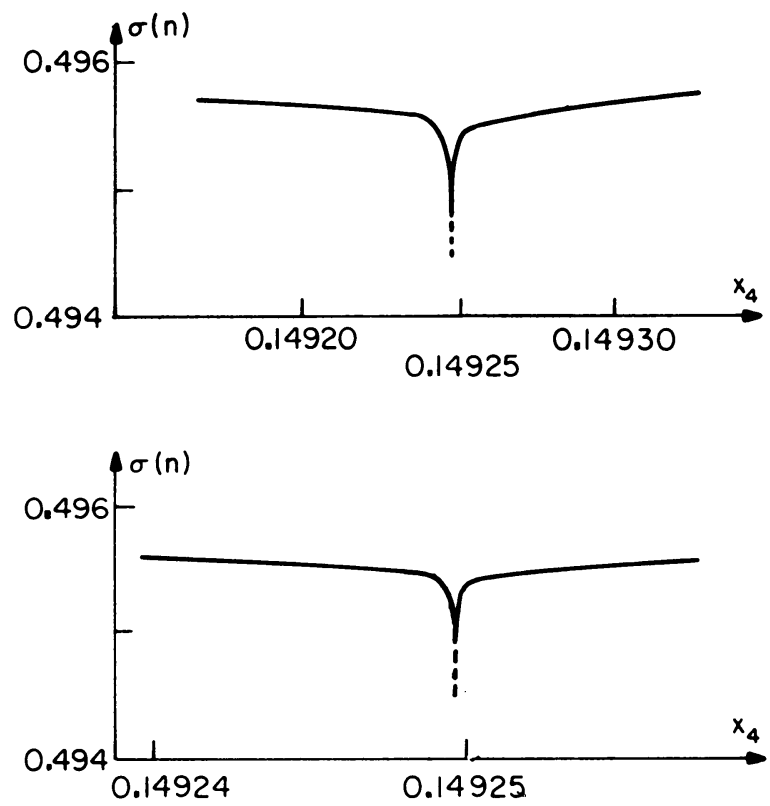

Figure 7

Minimization of $\sigma(n)$ for $x_{3}=1.168$ in two scales, computed values

--- extrapolated to $n \rightarrow \infty$. 
It is hopeless to minimize $S(\mathbf{x})$ as a function of over 500 variables $x_{i}$. But it is even difficult to find the minimum $\sigma=f\left(x_{3}, x_{4}\right)$ for two variables. This problem should represent a challenge to the existing minimization routines. The function $f\left(x_{3}, x_{4}\right)$ is easily evaluated, since it takes fewer than 25 short FORTRAN statements.

An explicit function which exhibits a similar behavior is for instance

$$
f(x, y)=\frac{10}{10+(x-1 / 2)^{6}}-10\left\{\log \left(y-\frac{1}{1+(10 x-5)^{4}}\right)^{2}\right\}^{-2}
$$

The exact maximum is $f(1 / 2,1)=1$, but it is located at a bend of a canyon with steep walls. Standard minimization routines might not find it easy to solve this problem.

Department of Mathematics

University of Southern California

Log Angeles, California 90007

1. D. E. DAYKIN, "Inequalities for functions of cyclic nature," J. London Math. Soc. (2), v. 3, 1971 , pp. 453-462.

2. P. H. DIANANDA, “On a cyclic sum," Proc. Glasgow Math. Assoc., v. 6, 1963, pp. $11-$ 13.

3. P. H. DIANANDA, "A cyclic inequality and an extension of it. II," Proc. Edinburgh Math. Soc. (2), v. 13, 1962/1963, pp. 143-152.

4. D. Ž. DJOKOVIĆ, "Sur une inégalité," Proc. Glasgow Math. Assoc., v. 6, 1963, pp. $1-10$.

5. D. S. MITRINOVIĆ, Analytic Inequalities, Springer-Verlag, New York, 1970, p. 132 ff.

6. P. NOWOSAD, "Isoperimetric eigenvalue problems in algebra," Comm. Pure Appl. Math., v. 21, 1968 , pp. $401-465$.

7. R. A. RANKIN, “An inequality," Math. Gaz., v. 42, 1958, pp. 39-40.

8. J. L. SEARCY \& B. A. TROESCH, "A cyclic inequality and a related eigenvalue problem," Pacific J. Math., v. 81, 1979, pp. 217-226.

9. H. S. SHARIRO, “Problem 4603," Amer. Math. Monthly, v. 61, 1954, p. 571. 